EPJ Web of Conferences 113,01007 (2016)

DOI: 10.1051/epjconf/201611301007

(C) Owned by the authors, published by EDP Sciences, 2016

\title{
Hadronic Weak Interaction Studies at the SNS
}

\author{
Nadia Fomin 1, a \\ ${ }^{1}$ University of Tennessee, Knoxville, TN 37996
}

\begin{abstract}
.
Neutrons have been a useful probe in many fields of science, as well as an important physical system for study in themselves. Modern neutron sources provide extraordinary opportunities to study a wide variety of physics topics. Among them is a detailed study of the weak interaction. An overview of studies of the hadronic weak (quark-quark) as well as semi-leptonic (quark-lepton) interactions at the Spallation Neutron Source (SNS) is presented. These measurements, done in few-nucleon systems, are finally letting us gain knowledge of the hadronic weak interaction without the contributions from nuclear effects. Forthcoming results from the NPDGamma experiment will, due to the simplicity of the neutron, provide an unambiguous measurement of the long range pion-nucleon weak coupling (often referred to as $h_{\pi}$ ), which will finally test the theoretical predictions. Results from NPDGamma and future results from the $n+{ }^{3} \mathrm{He}$ experiment will need to be complemented by additional measurements to completely describe the hadronic weak interaction.
\end{abstract}

\section{Introduction}

The hadronic weak interaction has been an object of experimental study for many decades, but it still holds many mysteries. While parity violation has been observed many times in nuclear systems, corrections for nuclear effects are required in order to isolate any $N N$ components. To avoid this, it is desirable to use the simplest systems available to conduct these studies. The hadronic weak interaction program at the SNS uses two of the smallest and well understood nuclei for this purpose.

The Fundamental Neutron Physics Beamline (FnPB) [1] at the SNS will be home to a series of experiments that aim to answer fundamental questions in nuclear physics, including studies of the hadronic weak interaction between nucleons. The NPDGamma and $n+{ }^{3} \mathrm{He}$ experiments are set to test the strength of the hadronic weak interaction between nucleons. This interaction was first parametrized in terms of meson exchange in the DDH model [2] over thirty years ago. However, the meson exchange couplings are still largely unknown today. Modern theoretical frameworks include Effective Field Theory [3] as well as Lattice QCD [4]. EFTs are model independent and are able to provide systematic theoretical error estimates, as well as a connection to QCD via symmetry principles. Additionally, EFTs are useful in interpreting experimental data and calculations of parityviolating observables in systems of up to five nucleons are currently possible. Lattice QCD is on its way to becoming a reliable tool for hadronic parity violation calculations, and future experiments present exciting opportunities to test precise predictions.

a e-mail: nfomin@utk.edu 
The first SNS experiment to take data is NPDGamma, which aims to measure a parity violating asymmetry in the direction of gamma rays emitted from cold neutron capture on parahydrogen [5, 6]. This asymmetry is directly related to the long range pion-nucleon weak coupling, $h_{\pi}^{1}$ in the DDH framework of meson exchange [2]. In the DDH model, physical observables can be written in terms of several total meson couplings, with several experiments necessary to isolate them all. In case of NPDGamma, the experimental observable is a parity violating asymmetry between the spin of the neutron and the direction of the outgoing gamma in cold neutron capture on hydrogen. In the DDH model, this asymmetry is given by:

$$
A_{\gamma}=-0.11 h_{\pi}^{1}-0.001 h_{\rho}^{1}+0.004 h_{\omega}^{1}
$$

where the subscripts reference the meson, and the superscript indicate the isospin change. The weak couplings ( $h$ 's) are expected to be of the same order, meaning that NPDGamma is primarily sensitive to the $h_{\pi}^{1}$ coupling, and isolates the $\Delta \mathrm{I}=1$ part of the hadronic weak interaction, unencumbered by nuclear effects. The DDH model predicts $h_{\pi}^{1}$ to be $\sim 4.5 \times 10^{-7}$, with a "reasonable range" of -1 to 11 $\left(\mathrm{x} 10^{-7}\right)$. The high flux at the FnPB has allowed for a measurement with $1.3 \times 10^{-8}$ precision.

A more modern approach to the problem is to use effective field theories [3] which are model independent and provide systematic theoretical error estimates. In effective field theory, the NPDGamma observable is given by [7]:

$$
A_{\gamma}=\frac{4}{3} \sqrt{\frac{2}{\pi}} \frac{M^{3 / 2}}{\kappa_{1}\left(1-\gamma a^{1} S_{0}\right)} g^{3} S_{1-{ }^{3} P_{1}},
$$

where all parameters are known experimentally, and a measurement of $A_{\gamma}$ will isolate $g^{3} S_{1}-{ }^{3} P_{1}$ low energy coupling constant. Finally, a first calculation of $h_{\pi}^{1}$ has been done on the lattice [4], with a result of $1.099 \pm 0.505_{-0.064}^{+0.058}\left[x 10^{-7}\right]$.

The $n+{ }^{3} \mathrm{He}$ experiment to search for a parity-odd asymmetry in the $\vec{n}+{ }^{3} \mathrm{He} \rightarrow \mathrm{p}+\mathrm{T}+765 \mathrm{keV}$ reaction is running at the Spallation Neutron Source. This is a natural follow-on to NPDGamma and is sensitive to $\Delta I=0,1$, and 2 parts of the hadronic weak interaction. The projected error on the asymmetry is $1.6 \times 10^{-8}$ and the experiment is expected to finish taking data in 2015 . Unlike NPDGamma, this asymmetry probes $\Delta I=0,1$, and 2 components of the NN weak interaction and needs additional DDH couplings to be described [8]:

$$
A_{p}=-0.185 h_{\pi}^{1}-0.038 h_{\rho}^{0}+0.023 h_{\rho}^{1}-0.001 h_{\rho}^{2}-0.050 h_{\omega}^{0}-0.023 h_{\omega}^{1} .
$$

The $\vec{n}+{ }^{3} \mathrm{He} \rightarrow p+t+765 \mathrm{keV}$ process has also been calculated in n chiral EFT [9].

\section{$2 \vec{n}+p \rightarrow d+\gamma:$ The NPDGamma experiment}

The NPDGamma experiment isolates the $\Delta \mathrm{I}=1$ part of the weak interaction by measuring the correlation between the neutron spin and the direction of the outgoing gamma in the capture of cold neutrons on protons. The deuteron is the most loosely bound of all nuclei, meaning the weak $N N$ interaction is dominated by the long-range component. The simple nature of the interaction also eliminates the need for any nuclear physics corrections, making the results easy to interpret.

The experimental apparatus is shown in Fig. 1. NPDGamma ran on the FnPB [10] at the SNS from 2011 until June, 2014. Cold neutrons were polarized via a supermirror located $\sim 15 \mathrm{~m}$ downstream of the moderator face. The neutron beam is pulsed at $60 \mathrm{~Hz}$, meaning that the neutron velocities and energies can be inferred from their times of flight. This allows for the spins of the neutrons to be reversed on a pulse-by-pulse basis through the use of an RF spin rotator [11]. The data were collected 


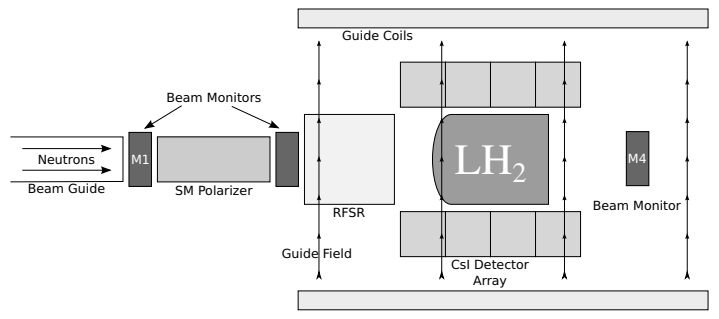

Figure 1. Layout of the NPDgamma apparatus. See text for details.

in spin octets ( $\downarrow \downarrow \uparrow \uparrow \downarrow \uparrow \uparrow \downarrow$ and $\downarrow \uparrow \uparrow \downarrow \uparrow \downarrow \downarrow \uparrow)$ in order to cancel any time-dependent systematic effects to the second order. The neutrons are then captured on a $16 \mathrm{~L}$ target made of parahydrogen at $\sim 16 \mathrm{~K}$. The gammas from the neutron capture on protons are detected via a $3 \pi$ acceptance detector array surrounding the hydrogen target. There are 48 detectors, arranged in 4 rings of 12 around the target. Each detector consists of 2 CsI crystals $\left(15.2 \times 15.2 \times 15.2 \mathrm{~cm}^{3}\right)$, viewed by a vacuum photodiode [12]. The neutron flux makes a counting experiment impractical, so the detectors are operated in currentmode. Finally, the experimental apparatus is held inside a $\sim 10$ Gauss magnetic field to maintain the neutron polarization on the way to the hydrogen target.

A raw asymmetry is formed for octets ( 8 accelerator pulses worth) of data, 4 of which will have had neutrons with spins aligned with the magnetic field and 4 anti-aligned. Asymmetries are formed between pairs of detectors at the same angle with respect to the magnetic field in order to cancel pulse-to-pulse beam fluctuations in the following manner:

$$
A_{\text {raw }}=\frac{\left(Y_{i}^{\uparrow}+Y_{i+6}^{\downarrow}\right)-\left(Y_{i}^{\downarrow}+Y_{i+6}^{\uparrow}\right)}{Y_{i}^{\uparrow}+Y_{i+6}^{\downarrow}+Y_{i}^{\downarrow}+Y_{i+6}^{\uparrow}},
$$

where the $Y$ 's are the yields in pairs of detectors.

Each detector has a sensitivity to the up-down and left-right asymmetries given by the so-called "geometric factors". This sensitivity depends on the position of the detector with respect to the point of neutron capture. The geometrical factors are average energy-weighted functions that are a measure of the emission direction of a photon from the target that deposits energy in a given detector. They were obtained by a combination of Monte Carlo techniques and measurements with a known ${ }^{137} \mathrm{Cs}$ source.

To extract the physics asymmetries $\left(A_{U D}\right.$ and $\left.A_{L R}\right)$, several corrections need to be made. Multiplicative corrections for the polarization of the incoming neutron beam, depolarization in the hydrogen target, the efficiency of the RF spin rotator are applied. In addition to capture on the parahydrogen, neutrons are also captured by the aluminum in the cryogenic target vessel. To account for this contribution to our detector signals, data were taken on a specially constructed aluminum target, and the up-down and left-asymmetries also determined. To extract the proton physics asymmetries, we fit the data to the following expression:

$$
\begin{array}{r}
A_{\text {raw }}[i]=\left(F_{H} \cdot A_{U D}^{H}[i] \cdot G_{U D}^{H}[i]+F_{H} \cdot A_{L R}^{H}[i] \cdot G_{L R}^{H}[i]+F_{A L} \cdot A_{U D}^{A L}[i] \cdot G_{U D}^{A L}[i]+\right. \\
\left.F_{A L} \cdot A_{L R}^{A L}[i] \cdot G_{L R}^{A L}[i]\right) \cdot C_{e f f}
\end{array}
$$

where $F_{H}$ and $F_{A L}$ are the prompt signal captures of neutron capture on hydrogen and aluminum for detector element $i, G_{X Y}$ are the geometric factors for detector element $i$ for the hydrogen and aluminum 


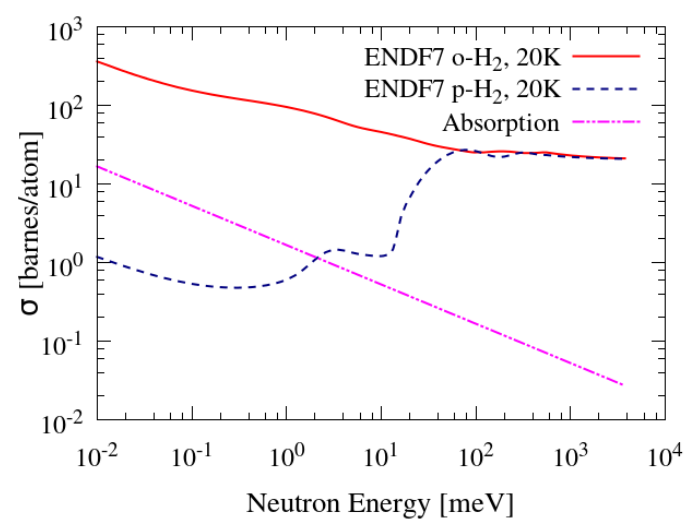

Figure 2. Neutron hydrogen scattering cross sections for ortho-hydrogen, para-hydrogen as well as absorption.

targets, and $C_{e f f}$ is the correction factor that's a product of the neutron beam polarization, RFSF efficiency, and the depolarization factor.

Neutron beam polarization and RFSF efficiency were measured in a separate experiment using transmission measurements through a polarized ${ }^{3} \mathrm{He}$ cell of both neutron spin states. Depolarization due to spin-flip scattering on hydrogen was calculated using Monte Carlo techniques and known scattering cross-sections. Fig. 2 shows the scattering cross sections on the two molecular states of hydrogen: ortho (proton spins aligned) and para (proton spins anti-aligned). Polarized neutrons that scatter on ortho-hydrogen depolarize before capture, which is the reason that the hydrogen target was operated at $<20 \mathrm{~K}$, where the admixture of orthohydrogen is very small at thermodynamic equilibrium. However, as hydrogen is circulated through the target vessel, some part of the volume may not be in thermodynamic equilibrium, and the maximum orthohydrogen concentration can be inferred from a cross section measurement recently performed [13] using the NPDGamma apparatus. Depolarization values were calculated using thermodynamic equilibrium and maximum orthohydrogen contamination scenarios. An average of the two values will be used as a correction in the final analysis.

The NPDGamma experiment completed its production data taking in June of 2014. There are three independent analysis of the hydrogen data being performed. They are in good agreement with each other as can be seen from raw detector asymmetries in Fig.3. While the parity violating asymmetry result is not yet available, the uncertainty on the measurement will be $<1.4 \times 10^{-8}$.

\section{$3 \vec{n}+{ }^{3} \mathrm{He} \rightarrow p+T+765 \mathrm{keV}:$ The $\mathbf{n}+{ }^{3}$ He experiment}

The study of the hadronic weak interaction continues at the FnPB at the SNS with the $n+{ }^{3} \mathrm{He}$ experiment, which aims to measure the directional asymmetry in the capture of cold neutrons on ${ }^{3} \mathrm{He}$. This asymmetry is sensitive to a combination of weak couplings, and will complement other measurements of the kind. The goal is achieve a combined statistical and systematic uncertainty of $<1.6 \times 10^{-8}$.

The experimental concept is similar to that of NPDGamma. The same supermirror polarizer is used to produce a polarized neutron beam. A new spin rotator has been constructed to allow the neutron spin to be rotated to point either along or against the direction of momentum. The spin flipper is a double cosine theta coil design providing a uniform interior transverse RF magnetic field oscillating at $\sim \mathrm{kHz}$. The neutrons are captured and detector in a target-detector wire chamber filled 


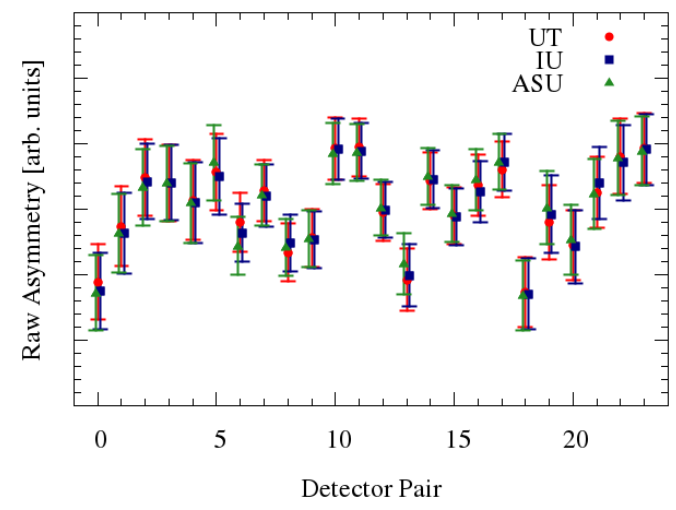

Figure 3. Raw hydrogen data asymmetries (arbitrary units) from three independent analyses.

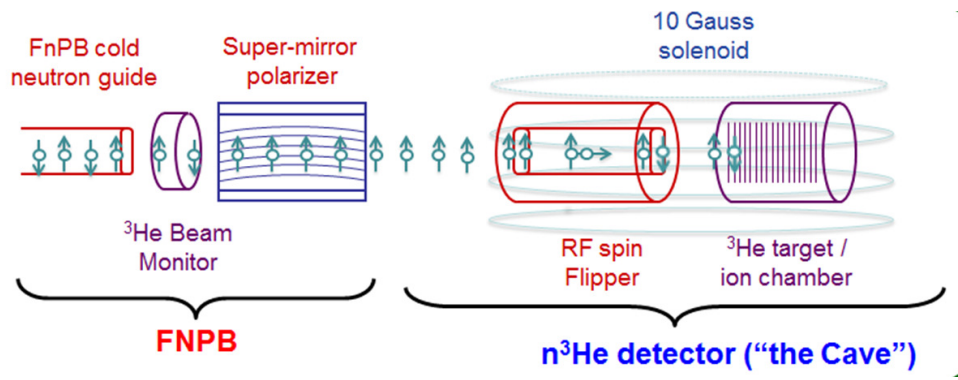

Figure 4. Experimental layout of the $n+3 H e$ apparatus. Details in text.

with ${ }^{3} \mathrm{He}$ gas so as to be completely absorbing. Neutrons that capture on ${ }^{3} \mathrm{He}$ produce an excited ${ }^{4} \mathrm{He}$ nucleus which then decays into a proton and a triton. Both the proton and triton then ionize the gas as they move through the gas volume and the resulting electrons are carried away by the anode wires. There are 17 high voltage frames with 8 wires each and 16 signal frames with 9 wires each, for a total of 144 signals to be read out. The range of the tritons $(\sim 1 \mathrm{~cm})$ is much smaller than that of the protons $(4 \mathrm{~cm})$, and most of the energy is carried away by the protons. As with NPDGamma, the detector is operated in current mode.

The ion chamber was commissioned in late 2014. The pulse integrals of the individual detector signal wire signals are shown in Fig. 5. As can be seen, most of the neutron beam is stopped in the first third of the wire chamber.

The $n+{ }^{3} \mathrm{He}$ experiment is to take production data until the end of 2015 calendar year to achieve its goal of $<1.6 \times 10^{-8}$ total uncertainty.

\section{Summary}

The FnPB at the SNS has been home to two important measurements of the hadronic weak interaction. NPDGamma's result will be the first measurement of the long-range $\Delta I=1$ component of the weak NN interaction that's free from nuclear uncertainties. The $n+{ }^{3} \mathrm{He}$ experiment continues this study as part 


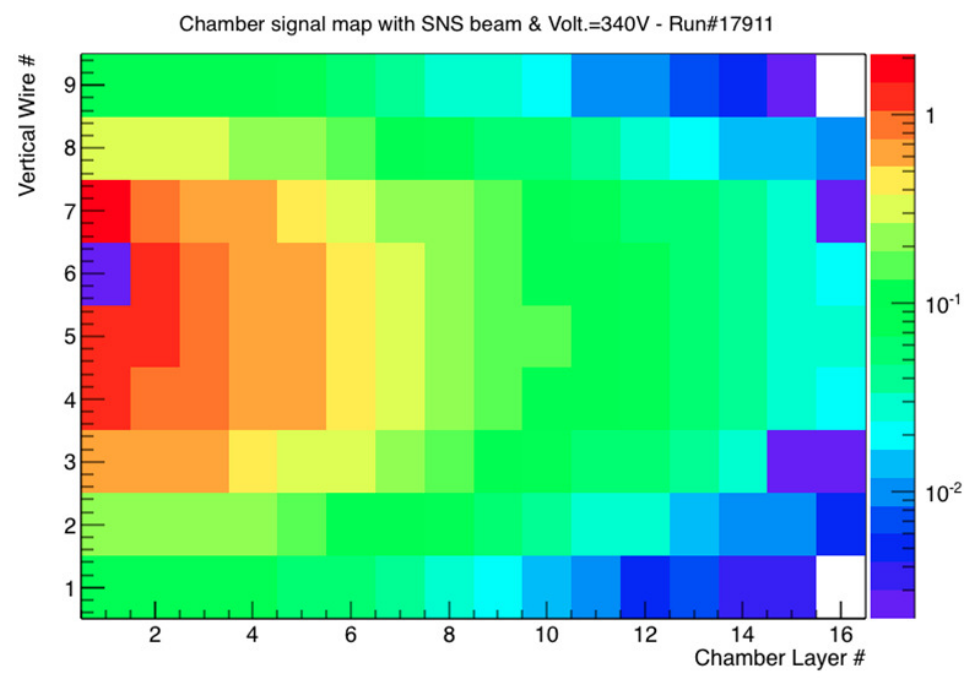

Figure 5. Integrated wire signals in the ion chamber.

of a larger effort in the field to finally pin down the weak couplings between nucleons after decades of measurements.

\section{References}

[1] N. Fomin and others, Nucl. Instr. Meth. A773 (2015) 45.

[2] B. Desplanques et al, Annals Phys. 124 (1980) 449.

[3] Shu-Lin Zhu et al, Nuc. Phys. A478 (2005) 435.

[4] J. Wasem, Phys. Rev. C 85, 022501 (2012).

[5] N. Fomin, AIP Conf. Proc. 1560 (2013) 145.

[6] M. Gericke et al, Phys. Rev. C 83, 055505 (2011).

[7] M. R. Schindler and R. P. Springer, Prog. Part. Nucl. Phys. 72, 1 (2013) [arXiv:1305.4190 [nuclth]].

[8] M. Viviani, R. Schiavilla, L. Girlanda, A. Kievsky and L. E. Marcucci, Phys. Rev. C 82, 044001 (2010)

[9] M. Viviani, A. Baroni, L. Girlanda, A. Kievsky, L. E. Marcucci and R. Schiavilla, Phys. Rev. C 89, 064004 (2014) doi:10.1103/PhysRevC.89.064004 [arXiv:1403.2267 [nucl-th]].

[10] N. Fomin et al., Nucl. Instrum. Meth. A 773, 45 (2014)

[11] P.-N. Seo et al., Phys. Rev. ST Accel. Beams 11, 084701 (2008) [arXiv:0710.2871 [nucl-ex]]

[12] M. T. Gericke et al., Nucl. Instrum. Meth. A 540, 328 (2005)

[13] K. B. Grammer et al., Phys. Rev. B 91, no. 18, 180301 (2015) 